\title{
Procedimiento para gestionar el Sistema DE PRODUCCIÓN EN EL SECTOR DE ALIMENTOS EN EMPRESAS CUBANAS
}

\author{
María de los Ángeles CAMPOS FERnÁNDEZ* \\ https://orcid.org/0000-0002-4591-8179 \\ Alexey Megna Alicio* \\ https://orcid.org/0000-0001-6714-0452 \\ Geinier Ramírez Camejo* \\ https://orcid.org/0000-0002-8281-7115 \\ Universidad de Las Tunas
}

Recibido: 22 de octubre del 2020 / Aceptado: 23 de noviembre del 2020

doi: https://doi.org/10.26439/ing.ind2021.n40.4897

RESUMEN: El objetivo del presente artículo lo constituye el diseño de un procedimiento para gestionar el sistema de producción, considerando las principales exigencias técnico-organizativas y político-económicas del proceso, así como sus factores claves y elementos esenciales. Se realizó un análisis exhaustivo acerca de las metodologías consultadas con variables de selección, obteniendo como resultado final el procedimiento propuesto, el cual aporta una vía para perfeccionar la planificación, la organización y el control de la producción en empresas productoras de alimentos.

PALABRAS CLAVE: procedimiento / sistemas de producción / gestión de producción

\section{A PROCEDURE TO MANAGE THE PRODUCTION SYSTEM IN CUBAN FOOD COMPANIES}

ABSTRACT: The objective of this article is to design a procedure to manage the production system, considering the main technical-organizational and political-economic requirements of the process, as well as its key factors and essential elements. An exhaustive analysis of the consulted methodologies was carried out using selection variables. It was concluded that the proposed procedure improves production planning, organization and control in food companies.

KEYWORDS: procedure / production systems / production management

*Correos electrónicos: mariacf@ult.edu.cu; alexeyma@ult.edu.cu; geinierrc@ult.edu.cu 


\section{INTRODUCCIÓN}

En la última década, se ha originado un notable incremento en la influencia del subsistema de producción, dentro del ámbito de desarrollo empresarial, convirtiéndose los sistemas de gestión de producción en factor clave para el desarrollo y el crecimiento de las producciones manufactureras, integrando las diferentes funciones de planificación y mando, a partir de la utilización de técnicas, diagramas, gráficos y tipos de software que facilitan los cálculos y decisiones en torno a la selección de las mejores variantes de producción. En este caso, resulta válido resaltar que los niveles de planificación, ejecución y control se encuentran estructurados en un orden jerárquico que contemplan tanto los planes agregados y maestros, como la gestión de materiales, los niveles de ejecución, entre otros (Sipper y Bulfin Jr., 2009).

Estos sistemas deben estar dirigidos, principalmente, hacia el logro de los objetivos de la organización (obtener beneficios, satisfacer al cliente, tanto en plazos como en calidad, obtener producción al más bajo costo y con el menor consumo de material posible). Teniendo en cuenta que la gestión de la producción constituye el elemento esencial para aumentar la competitividad dentro de las empresas, proyecta, además, una imagen de calidad, con una mejor planificación y la disminución del nivel de existencias.

La efectividad de las medidas, los métodos y los procedimientos se logra cuando se adopta un sistema con criterio en su formulación y, a su vez, con la máxima objetividad. Esto último se logra con un dominio teórico y práctico de las principales bases metodológicas de gestión de producción (el sujeto de la gestión de la producción, las exigencias técnico-organizativas, los principios, los elementos, el método de estudio y el sistema de producción como objeto de la gestión de producción) (Acevedo Suárez, Gómez Acosta, Urquiaga Rodríguez y Hernández Torres, 2002).

\footnotetext{
El dominio de estas bases metodológicas es esencial para poder dirigir el perfeccionamiento de la gestión de producción en la empresa. Este perfeccionamiento abarca tanto el aspecto operativo como perspectivo. En el primero debe ajustarse o corregirse la organización del sistema productivo acorde con la situación y concreción de las exigencias del momento. El perfeccionamiento perspectivo requiere el estudio del desarrollo y cambios organizativos a realizar en el sistema productivo para su adaptación a las mismas. (Acevedo Suárez et al., 2002)
}

Cuba ha tenido que enfrentar la inestabilidad en los precios de los productos a adquirir en el mercado internacional, el incremento de las restricciones en las posibilidades de obtener financiamiento externo y el recrudecimiento del bloqueo económico, comercial y financiero impuesto por los Estados Unidos. Por lo que incrementar al máximo la eficiencia y competitividad constituyen grandes retos a alcanzar por las empresas cubanas para poderse integrar en el mundo empresarial contemporáneo, caracterizado por la imposición de requerimientos cada vez más exigentes. Para lograr esto, los cuadros de dirección de las empresas deben desplegar una ardua labor en la 
búsqueda y aplicación de formas y métodos novedosos para proporcionar una respuesta positiva ante las limitaciones del entorno.

A partir del VI Congreso del Partido Comunista de Cuba, realizado entre los días 16 y 19 de abril de 2011, en La Habana, el país emprendió la actualización de su modelo económico, lo cual ha demandado conformar un nuevo escenario para el desempeño de las entidades. El actual modelo económico cubano demanda que estas dediquen sus mayores esfuerzos a la búsqueda e implementación de alternativas para elevar al máximo sus resultados, para lo cual es imprescindible una adecuada gestión de sus procesos productivos.

En el año 2016, se celebró por primera vez en Cuba la V Conferencia Mundial de Gestión de Producción y Operaciones, con el objetivo de que los cubanos se integraran a la red mundial, adquiriendo conocimientos que les permitiera fortalecer este aspecto dentro de las empresas y, por consiguiente, en la economía cubana.

Según el Decreto Ley sobre la Continuidad y Fortalecimiento del Sistema de Dirección y Gestión Empresarial Cubano (Decreto Ley n. ${ }^{\circ} 252,2013$ ), concede gran importancia a la producción de bienes y a la prestación de servicios con la calidad requerida, en el momento adecuado y con la mayor eficiencia económica, en correspondencia con el objeto empresarial aprobado a la empresa u organización superior de dirección, a partir de las necesidades planteadas por la sociedad y los clientes.

Resulta imprescindible avanzar en el logro del ciclo completo de producción, mediante los encadenamientos productivos entre organizaciones que desarrollan actividades productivas, incluidas las universidades, para garantizar el desarrollo rápido y eficaz de nuevos productos y servicios. Así como incorporar los resultados de la ciencia, la innovación y la tecnología en los procesos productivos y de servicios; adoptar nuevos patrones de utilización de los factores productivos, modelos gerenciales y de organización de la producción; y concluir el proceso de reordenamiento y reestructuración del sistema empresarial, incluyendo el paso a nuevas formas de gestión (Partido Comunista de Cuba, 2016).

En los últimos tiempos, han existido irregularidades dentro de la cooperación entre las subdivisiones productivas de la empresa objeto de investigación, que limitan el funcionamiento de un proceso productivo integrado, influyendo negativamente en el cumplimiento de sus planes de producción anuales. Además de existir poca capacidad de diagnóstico y falta de empleo sistemático de herramientas analíticas que expliquen las causas del comportamiento de sus procesos, lo que se manifiesta en la repetición anual de los problemas.

Un sistema productivo que pretenda ser competitivo en este entorno tiene que poseer ciertas características, que en otras condiciones no eran tan importantes. Aspectos como la flexibilidad, adaptabilidad y rapidez de respuesta han pasado de ser aspectos deseables, a convertirse en la clave del éxito de muchas empresas (Araúzo Araúzo, de Benito Martín, del Olmo Martínez y Sanz Angulo, 2004). 
Un sistema productivo es una entidad que está dotada de un sistema de recursos, los que deben ser transformados en determinados resultados, que satisfagan las exigencias y requerimientos de los clientes. Para lograr dicha transformación, los sistemas productivos adoptan determinadas formas en su funcionamiento, conocidas como tipos de sistemas productivos (Acevedo Suárez et al., 2002). Definición asumida por los autores, puesto que esclarece que un sistema productivo es una entidad que gestiona las actividades del sistema, contando con recursos materiales, humanos y políticos, cuyos resultados están dirigidos a satisfacer la demanda con la calidad y eficiencia requerida.

Esta situación llevó a los autores a plantearse cómo mejorar el sistema de producción dentro de las empresas manufactureras, específicamente, en la de alimentos, aspecto que contribuye a la mejora de la eficiencia y eficacia con que se realizan las producciones.

La significación práctica radica en que, respecto al tema, hay insuficientes procedimientos específicos de gestión de producción, siendo los existentes muy abarcadores de todos los procesos de las empresas, por lo que el propuesto, en el presente artículo, considera las principales exigencias técnico-organizativas y político-económicas del proceso, así como sus factores clave y elementos esenciales.

\section{MÉTODOS DE INVESTIGACIÓN TECNOLÓGICA APLICADA}

Se consultaron procedimientos relacionados con el estudio de la gestión de la producción, entre ellos la metodología para el estudio de la organización de los procesos citada por Batista (2007) y el método de estudio de la gestión de producción de Acevedo Suárez et al. (2002), por otro lado, existen procedimientos para la evaluación de la gestión productiva en determinados entornos de fabricación (Fundora Miranda, Taboada Rodríguez, Cuellar, Urquiaga Rodríguez y Sánchez Lara, 1987). Otros autores, como Portuondo Pichardo (1990), Ballou (1991) y Lefcovich (2004), proponen una serie de medidas que contribuyen a la comprobación de determinados aspectos específicos de la producción. Aunque es importante resaltar que, de todos los procedimientos consultados, el más integral, en materia de gestión de producción, es el método de estudio propuesto por Acevedo Suárez et al. (2002), aun cuando no se define la forma de selección del sistema de gestión de producción ni identifica las herramientas a utilizar con una aplicación lo suficientemente clara, para que permita a los directivos de las organizaciones su puesta en práctica.

Se definieron cinco variables, las cuales fueron consultadas a un grupo de expertos logrando una concordancia del 95 \%. Estas variables son: tipo de producción, exigencias técnico-organizativas, determinación de la capacidad de producción, análisis de los elementos de gestión de producción y selección del sistema de gestión de la producción acorde a los requerimientos de producción. Solamente los procedimientos de Batista (2007) y Acevedo Suárez et al. (2002) cumplieron con tres de las variables, mientras que 
el resto solo se enfocaban en elementos específicos de la gestión de producción orientados a la logística del proceso o a la calidad de este.

Se utilizó el método de expertos (seleccionados por el método Delphi), teniendo en cuenta que la cantidad de expertos depende de la complejidad y las características del trabajo a realizar. El grupo de expertos debe estar entre siete y quince para mantener un nivel de confianza y calificación elevado. Se debe elaborar una lista inicial de personas posibles de cumplir los requisitos para ser expertos en la materia a trabajar y realizar una valoración sobre el tipo de experiencia, evaluando de esta forma los niveles de conocimiento que poseen sobre la materia.

Para ello, la primera pregunta, realizada a dichos expertos, debe ser una autoevaluación sobre los niveles de información y argumentación que poseen sobre el tema en cuestión. Para el cálculo del coeficiente de conocimiento $(K c)$ se tiene en cuenta el rango seleccionado por el experto $j(n)$, calculando de la siguiente manera:

$$
K_{c j}=n(0,1)
$$

Los aspectos que influyen sobre el nivel de argumentación o fundamentación del tema a estudiar permiten calcular el coeficiente de argumentación (Ka) de cada experto, mediante la siguiente ecuación, donde $n_{i}$ es el valor correspondiente a la fuente de argumentación:

$$
K_{a}=\sum_{i=1}^{6} n_{i}
$$

En consecuencia, se propone un procedimiento general que integra estos aspectos (evaluación, selección y control). Para la selección del sistema de gestión de producción adecuado, la metodología propuesta por Cespón Castro, Ibarra Mirrón y Marrero Delgado (septiembre de 2005) resulta, a criterio de los autores, la más acertada.

\section{RESULTADOS}

\subsection{Procedimiento}

El procedimiento diseñado está estructurado en nueve pasos organizados en cuatro fases (preparación de las condiciones, análisis de la organización de la producción, búsqueda de alternativas y programa de mejora) como se muestra a continuación.

\subsubsection{Fase 1. Preparación de las condiciones}

El principal objetivo es llevar a cabo la caracterización de la organización, desde el punto de vista funcional y teniendo en cuenta los tres elementos fundamentales del sistema de producción: fuerza de trabajo, medios de trabajo y objeto de trabajo. Las principales técnicas a utilizar son la observación directa y el estudio de documentación. 
- Paso 1. Caracterización de la entidad objeto de estudio

Este paso es de mucha utilidad cuando el procedimiento es llevado a cabo por investigadores externos. Se proponen utilizar los elementos brindados por Pérez Campaña (2005a): nombre de la empresa, fecha de creación, ubicación, subordinación, objeto social, principales salidas y sus clientes, principales entradas y sus proveedores, principales recursos de que dispone y la clasificación atendiendo a diferentes criterios.

\begin{tabular}{|c|c|c|c|c|c|}
\hline Elementos & \multicolumn{5}{|c|}{ Variantes de clasificación } \\
\hline Tamaño & Grande & \multicolumn{2}{|c|}{ Mediana } & \multicolumn{2}{|c|}{ Pequeña } \\
\hline \multirow{2}{*}{$\begin{array}{l}\text { Forma de } \\
\text { propiedad }\end{array}$} & \multicolumn{2}{|c|}{ Privadas } & \multirow{2}{*}{ Estatales } & \multirow{2}{*}{\multicolumn{2}{|c|}{ Mixta }} \\
\hline & Individuales & Sociedades & & & \\
\hline Sector genérico & \multicolumn{3}{|c|}{ Producción } & \multicolumn{2}{|c|}{ Servicios } \\
\hline $\begin{array}{l}\text { Tipo de } \\
\text { producción }\end{array}$ & Masiva & \multicolumn{2}{|c|}{ Seriada } & \multicolumn{2}{|c|}{ Unitaria } \\
\hline $\begin{array}{l}\text { Actividad } \\
\text { principal }\end{array}$ & Producción & Almacenamiento & Transportación & $\begin{array}{l}\text { Intercambio } \\
\text { o suministro }\end{array}$ & $\begin{array}{l}\text { Servicio } \\
\text { (sentido } \\
\text { estrecho) }\end{array}$ \\
\hline $\begin{array}{l}\text { Tipo del flujo } \\
\text { del proceso }\end{array}$ & Línea & \multicolumn{2}{|c|}{ Intermitente } & \multicolumn{2}{|c|}{ Por proyecto } \\
\hline $\begin{array}{l}\text { Forma del } \\
\text { pedido }\end{array}$ & \multicolumn{3}{|c|}{ Por pedido } & \multicolumn{2}{|c|}{ Para inventario } \\
\hline \multirow{2}{*}{$\begin{array}{l}\text { Relación pro- } \\
\text { ductor-cliente }\end{array}$} & \multirow{2}{*}{$\begin{array}{c}\text { Contra } \\
\text { almacén }\end{array}$} & \multicolumn{4}{|c|}{ Entrega directa } \\
\hline & & \multicolumn{2}{|c|}{ Con cobertura } & \multicolumn{2}{|c|}{ Sin cobertura } \\
\hline \multirow{2}{*}{$\begin{array}{l}\text { Forma de eje- } \\
\text { cutar el pedido }\end{array}$} & \multirow{2}{*}{ Por ritmo } & \multicolumn{3}{|c|}{ Programado } & \multirow{2}{*}{ Por pedido } \\
\hline & & Cantidad fija & Frecuencia fija & Irregular & \\
\hline $\begin{array}{l}\text { Elemento a } \\
\text { optimizar }\end{array}$ & Ciclo & Materia prima & $\begin{array}{l}\text { Recursos } \\
\text { humanos }\end{array}$ & Capacidad & Energía \\
\hline Tipo de servicio & $\begin{array}{l}\text { Servicio en } \\
\text { masa }\end{array}$ & $\begin{array}{c}\text { Servicios } \\
\text { profesionales }\end{array}$ & $\begin{array}{l}\text { Fábrica de } \\
\text { servicio }\end{array}$ & \multicolumn{2}{|c|}{ Taller de servicio } \\
\hline \multicolumn{6}{|c|}{ Comercio minorista } \\
\hline \multirow{3}{*}{$\begin{array}{l}\text { Área destinada } \\
\text { a la venta y } \\
\text { productos que } \\
\text { oferta }\end{array}$} & Más de $350 \mathrm{~m}^{2}$ & Entre $100-350 \mathrm{~m}^{2}$ & Menor de $100 \mathrm{~m}^{2}$ & \multirow{2}{*}{\multicolumn{2}{|c|}{$\begin{array}{l}\text { Surtido extenso y profundo } \\
\text { de uno o varios departa- } \\
\text { mentos, con independen- } \\
\text { cia de los metros } \\
\text { cuadrados }\left(\mathrm{m}^{2}\right)\end{array}$}} \\
\hline & $\begin{array}{l}\text { Surtido exten- } \\
\text { so y profundo }\end{array}$ & $\begin{array}{l}\text { Surtido limitado y } \\
\text { poco profundo }\end{array}$ & $\begin{array}{l}\text { Surtido limitado } \\
\text { y poco profundo }\end{array}$ & & \\
\hline & $\begin{array}{c}\text { Por } \\
\text { departamentos }\end{array}$ & Mixta & $\begin{array}{c}\text { Conveniencia o } \\
\text { cercanía }\end{array}$ & \multicolumn{2}{|c|}{ Especializada } \\
\hline
\end{tabular}

Figura 1. Criterios de clasificación de las empresas

Fuente: Pérez Campaña (2005a) 
- Paso 2. Creación del grupo de trabajo

Consiste en trabajar y motivar a las personas implicadas en el proceso, dándole participación plena en la búsqueda de soluciones con un solo objetivo: la mejora continua de sus procesos de producción. En este caso, el grupo de trabajo se debe encontrar integrado por miembros del área de producción, calidad, seguridad y salud del trabajo y recursos humanos, con ayuda del investigador (principal responsable).

\subsubsection{Fase 2. Análisis de la organización de la producción}

Se declara esta fase con el objetivo de analizar las bases metodológicas que, según Acevedo Suárez et al. (2002), sustentan la gestión de la producción. En el caso específico del procedimiento propuesto para la industria cárnica, los elementos esenciales a evaluar son los que se reflejan en los pasos de esta fase. Las principales herramientas a utilizar son las entrevistas, las herramientas estadísticas, el algoritmo para el cálculo de la capacidad de producción y el análisis de indicadores de desempeño.

- Paso 3. Exigencias técnico-organizativas

Para desarrollar la gestión de producción, se hace imprescindible conocer cuáles son las exigencias que le plantea el medio al sistema productivo en cada momento y cuál es la organización interna que debe adoptar dicho sistema para lograr su máxima satisfacción. En este caso, se proponen cinco exigencias fundamentales propuestas por Torres Cabrera y Urquiaga Rodríguez (2007):

- Capacidad de reacción, cuyo principal indicador es el tiempo medio de reacción:

$$
\operatorname{Tr}_{\propto}=t_{\text {med }}+\hat{b} * \sigma
$$

- Dinámica de rendimiento, que exige que la organización de la producción adoptada garantice un crecimiento sistemático de los indicadores de eficiencia.

- Estabilidad, que demanda adoptar una organización de la producción que permita prever y resolver los problemas que surgen sin necesidad de la intervención de los niveles superiores. Su principal indicador se haya relacionado a la producción promedio por intervalo y la desviación típica de esa producción promedio, como se muestra en la ecuación (2):

$$
E s=1-\frac{\sigma}{\bar{X}}
$$


- Fiabilidad, que requiere el funcionamiento del sistema de producción durante un largo período sin afectaciones en las variables de resultado.

- Flexibilidad, que exige que la organización de la empresa asuma los cambios de producción en poco tiempo y a bajos costos.

- Paso 4. Principios de la gestión de producción

Expresan las reglas prácticas para adoptar una racional organización interna del sistema productivo para lograr la máxima eficiencia económica y social en el marco de la máxima satisfacción de las exigencias del medio. En este caso, se evaluarán tres principios fundamentales: proporcionalidad, ritmicidad y continuidad:

- Proporcionalidad: expresa la correspondencia necesaria entre las capacidades de producción de las subdivisiones productivas vinculadas entre sí en un mismo proceso, flujo o ruta tecnológica. Es la condición necesaria para lograr un adecuado nivel de la organización de la producción. Presupone la eliminación de los cuellos de botella. Su principal indicador se calcula de la siguiente forma:

$$
K p=1-\frac{\sum_{i=1}^{N}(X \max -X i) n_{i}}{N t * X \max }
$$

- Ritmicidad: se manifiesta en la obtención de igual volumen de producción en iguales intervalos de tiempo o en el cumplimiento de las tareas previstas según un programa de producción elaborado de antemano:

$$
K r=1-\frac{\sigma}{\bar{X}}
$$

- Continuidad: presupone el flujo del objeto de trabajo a lo largo de todo el proceso de producción sin interrupciones, así como la utilización plena de la fuerza de trabajo y los medios de trabajo. Es decir, la utilización continuada de los tres elementos del proceso de producción:

$$
\begin{gathered}
K c f=\frac{\sum_{f=1}^{S} T f}{\sum_{f=1}^{S} F T f} \\
K t m=\frac{\sum_{f=1}^{S} T m}{\sum_{f=1}^{S} F T m} \\
K c o=\frac{T t e c}{T c}
\end{gathered}
$$


- Paso 5. Elementos de la gestión de producción

En este paso se deben tener en cuenta los factores o elementos que influyen directamente en el sistema de producción (las formas de gestión, el método de producción, la gestión del flujo material, la gestión de la calidad y las normas del sistema de producción, lo que permite evaluar el cumplimiento de la organización establecida y fijar los objetivos en la elaboración de los planes).

- Paso 6. Capacidad de producción

La gestión de la capacidad asegura la máxima utilización de las materias primas y los equipos para alcanzar el volumen de producción que puede producir una instalación en determinado período de tiempo, además de determinar de forma más específica el punto limitante del proceso. En este caso, se recomienda el algoritmo propuesto por Torres Cabrera y Urquiaga Rodríguez (2007).

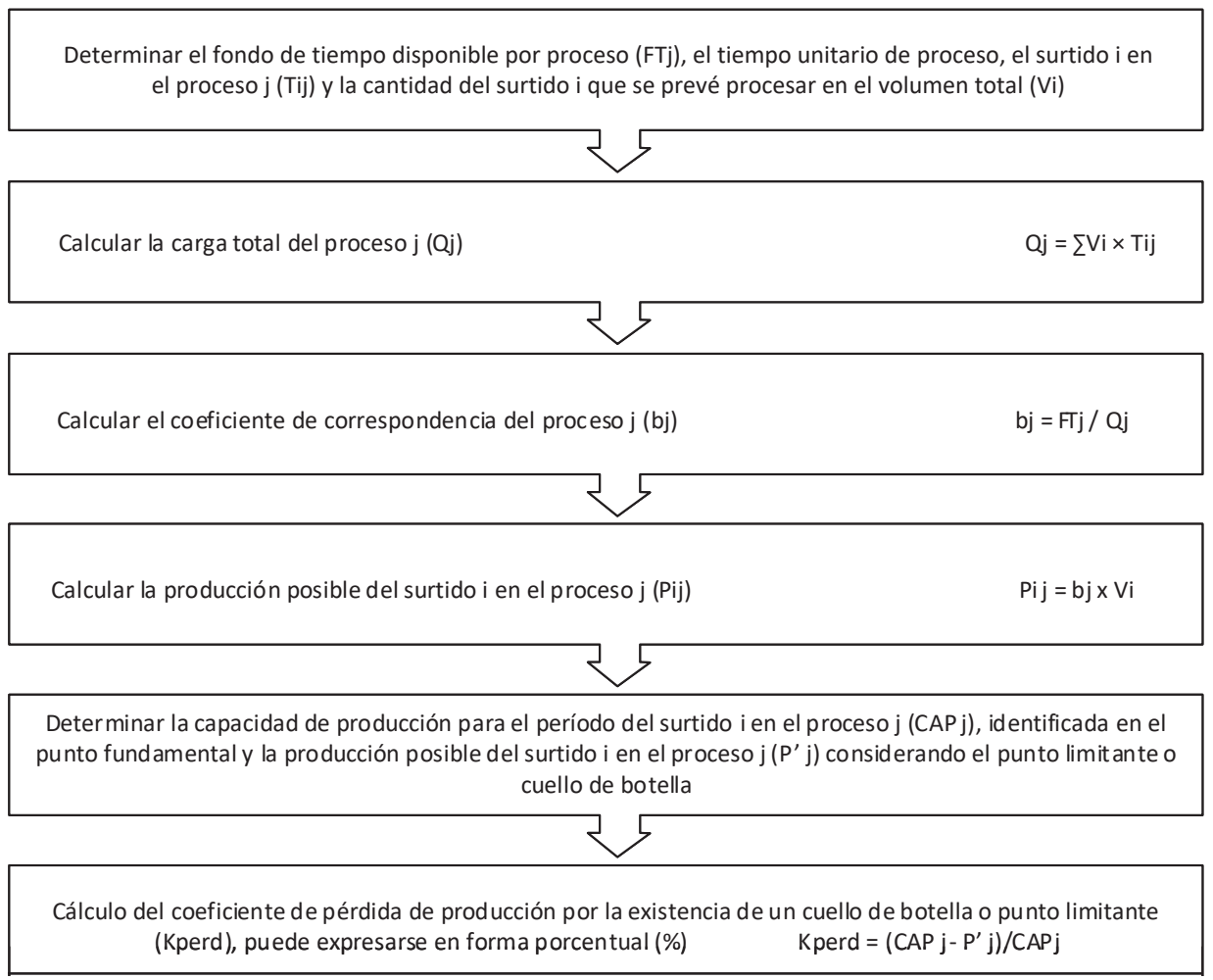

Figura 2. Algoritmo general para el cálculo de la capacidad de producción Fuente: Torres Cabrera y Urquiaga Rodríguez (2007) 


\subsubsection{Fase 3. Búsqueda de alternativas}

El principal objetivo de la presente fase radica en seleccionar el tipo de sistema de gestión de la producción que más se ajuste a las características del proceso y a las condiciones en que este opera. Las principales herramientas son la metodología para la selección del sistema de gestión de la producción y la revisión de documentos.

- Paso 7. Determinación de los sistemas de gestión de producción que se encuentren en concordancia con el sistema de producción en cuestión

Para este paso se propone el procedimiento para la determinación del tipo de sistema de gestión de la producción, elaborado por Cespón Castro et al. (septiembre de 2005), ya que ha sido aplicado con anterioridad en empresas cubanas con resultados favorables.

- Paso 8. Evaluación y selección

El objetivo fundamental de esta fase radica en la selección específica del tipo de sistema de gestión de la producción, apoyándose en los criterios establecidos previamente. Una vez conocidos los posibles sistemas de gestión de producción a implantar, se deben tener en cuenta los resultados obtenidos en la primera fase y los requisitos de aplicación de los sistemas seleccionados, con el fin de determinar el sistema más factible a implementar.

\subsubsection{Fase 4. Programa de mejora}

Se elabora el plan de acción para la aplicación del sistema seleccionado, evaluando cada uno de los elementos necesarios para su aplicación y se controla la correcta puesta en funcionamiento del mismo, con el fin de ajustar cualquier deficiencia detectada a partir de un nuevo diagnóstico. Las principales herramientas son el trabajo en equipo y los gráficos de Gantt.

\subsection{Validación a través del método de expertos}

Con el objetivo de validar la factibilidad del procedimiento diseñado, se decidió aplicar el método de especialistas, con base en el método de expertos (seleccionados por el método Delphi), lográndose la aprobación en la primera ronda de evaluación.

En el presente estudio, se seleccionaron nueve especialistas de las diferentes áreas administrativas de la empresa Cárnica Las Tunas, entidad donde se procederá a aplicar el procedimiento, cuyas funciones y responsabilidades se encuentran en correspondencia con los elementos de la gestión y de los sistemas de producción. Se les aplicó una encuesta con el objetivo de conocer el nivel de conocimientos que poseían respecto al tema abordado, cuyos resultados se muestran en la tabla 1. 
Tabla 1

Determinación del coeficiente de conocimiento

\begin{tabular}{|c|c|c|c|c|c|c|c|c|c|c|c|}
\hline \multirow{2}{*}{$\begin{array}{l}\text { Expertos } \\
\text { propuestos }\end{array}$} & \multicolumn{10}{|c|}{ Autoevaluación del nivel de conocimiento sobre el tema } & \multirow{2}{*}{$\begin{array}{l}\text { Kc de los } \\
\text { expertos } \\
\text { propuestos }\end{array}$} \\
\hline & 1 & 2 & 3 & 4 & 5 & 6 & 7 & 8 & 9 & 10 & \\
\hline 1 & & & & & & & & & & $x$ & 1,0 \\
\hline 2 & & & & & & & & & & $x$ & 1,0 \\
\hline 3 & & & & & & & & & $x$ & & 0,9 \\
\hline 4 & & & & & & & & & & $x$ & 1,0 \\
\hline 5 & & & & & & & & $x$ & & & 0,8 \\
\hline 6 & & & & & & $x$ & & & & & 0,6 \\
\hline 7 & & & & & & & $x$ & & & & 0,7 \\
\hline 8 & & & & & & & & & & $x$ & 1,0 \\
\hline 9 & & & & & & & & & $x$ & & 0,9 \\
\hline
\end{tabular}

Elaboración propia

Para determinar el coeficiente de argumentación de los especialistas, se valoraron aspectos como su conocimiento acerca de la entidad, años de experiencia, conocimientos teóricos y sus posibilidades de identificación de las fortalezas y debilidades de la gestión de la producción y los sistemas de producción. Cada especialista se autoevaluó y se clasificaron los resultados en tres niveles (alto, medio y bajo). En la tabla 2 se muestra el patrón de comparación utilizado para evaluar el coeficiente de argumentación de los expertos propuestos $(K a)$, donde se le atribuye a cada categoría una puntuación según su grado de importancia e influencia. Los resultados finales se muestran en la tabla 3.

Tabla 2

Patrón utilizado para el coeficiente de argumentación (Ka)

\begin{tabular}{lcccc}
\hline $\begin{array}{l}\text { Fuentes de argumentación } \\
\text { o fundamentación }\end{array}$ & Código & Alto & Medio & Bajo \\
\hline Conocimientos de la entidad & A & 0,5 & 04 & 0,3 \\
Años de experiencia & B & 0,3 & 0,2 & 0,1 \\
Conocimientos teóricos & C & 0,1 & 0,1 & 0,05 \\
Posibilidades de deter- & D & 0,1 & 0,1 & 0,05 \\
minación de fortalezas y & & & & \\
debilidades & & & & \\
\hline
\end{tabular}

Elaboración propia 
Tabla 3

Coeficiente de argumentación de cada experto

\begin{tabular}{cccccc}
\hline \multirow{2}{*}{ Expertos } & \multicolumn{4}{c}{ Autoevaluación de las fuentes de argumentación } & \multirow{2}{*}{ Ka } \\
\cline { 2 - 5 } & $\mathrm{A}$ & $\mathrm{B}$ & $\mathrm{C}$ & $\mathrm{D}$ & \\
\hline 1 & 0,5 & 0,3 & 0,05 & 0,1 & 0,95 \\
2 & 0,5 & 0,3 & 0,05 & 0,1 & 0,95 \\
3 & 0,5 & 0,3 & 0,1 & 0,1 & 1,0 \\
4 & 0,4 & 0,3 & 0,1 & 0,1 & 0,9 \\
5 & 0,5 & 0,3 & 0,1 & 0,1 & 1,0 \\
6 & 0,5 & 0,3 & 0,1 & 0,1 & 1,0 \\
7 & 0,5 & 0,3 & 0,1 & 0,1 & 1,0 \\
8 & 0,3 & 0,1 & 0,1 & 0,1 & 0,6 \\
9 & 0,5 & 0,3 & 0,1 & 0,1 & 1,0 \\
\hline
\end{tabular}

Elaboración propia

Teniendo en cuenta el coeficiente de argumentación (Ka) y el coeficiente de conocimiento $(K c)$, se determinó el coeficiente de competencia con el cual quedó determinado el grupo de expertos, teniendo en cuenta que el valor de este último debe ser siempre superior a 0,5, para que el especialista pueda formar parte de este grupo.

Tabla 4

Coeficiente de competencia de cada experto

\begin{tabular}{|c|c|c|c|c|c|c|c|c|c|}
\hline \multirow{2}{*}{ Coeficientes } & \multicolumn{9}{|c|}{ Expertos propuestos } \\
\hline & 1 & 2 & 3 & 4 & 5 & 6 & 7 & 8 & 9 \\
\hline$K c$ & 1 & 1 & 0,9 & 1 & 0,8 & 0,6 & 0,7 & 1 & 0,9 \\
\hline$K a$ & 0,95 & 0,95 & 1 & 0,9 & 1 & 1 & 1 & 0,6 & 1 \\
\hline K & 0,975 & 0,975 & 0,95 & 0,95 & 0,9 & 0,8 & 0,85 & 0,8 & 0,95 \\
\hline
\end{tabular}

Elaboración propia

En este caso, la selección de los expertos quedó en el número original. Al aplicar, entonces, la encuesta de factibilidad de la propuesta diseñada por los autores, se tuvieron en cuenta los siguientes indicadores cualitativos:

- Necesidad de utilizar una vía para perfeccionar la gestión y los sistemas de producción dentro de los procesos de cárnicos.

- Adaptabilidad del procedimiento a las características tecnológicas dentro de la entidad. 
- Secuencia lógica del procedimiento.

- Factibilidad de aplicación del procedimiento.

- Posibilidades de generalizar la Unidad Empresarial de Base (UEB).

- Utilidad para perfeccionar los sistemas de producción dentro de la Unidad Empresarial de Base (UEB).

El resultado final de la encuesta arrojó resultados satisfactorios, teniendo en cuenta que los expertos en su totalidad mostraron aceptación por el procedimiento propuesto.

Los resultados fueron los siguientes:

- El 67 \% consideró muy necesario perfeccionar el sistema de producción en la empresa, mientras que el $33 \%$ solo lo vio como simplemente necesario.

- El $78 \%$ de los encuestados coincidió en que el procedimiento propuesto consideró las características físicas y tecnológicas de la entidad, asimismo el 89 \% está de acuerdo en que las fases declaradas dentro del procedimiento se encontraban estructuradas con una lógica comprensible. Finalmente, se obtuvo que el $78 \%$ opina que sería factible aplicar el procedimiento.

- El total de los encuestados valoró que es posible aplicar el procedimiento en todas las UEB pertenecientes a la empresa.

- El procedimiento es válido para el diagnóstico de la organización de la producción (89\%).

- El $56 \%$ de los encuestados opinó que la aplicación de un nuevo sistema contribuye a perfeccionar la gestión de la producción parcialmente; mientras el $44 \%$ consideró que lo hará en su totalidad.

- Dentro de los beneficios que se estimaron en la aplicación del procedimiento, se encontraron los siguientes: lograr un máximo aprovechamiento de la mano de obra y de los medios de trabajo, identificar y erradicar los eslabones débiles dentro de la cadena productiva, aumentar la productividad del trabajo, elevar los principales indicadores de la empresa, disminuir los costos de producción, aumentar la capacidad productiva y elevar la eficiencia y la eficacia de la entidad.

\section{DISCUSIÓN}

En el año 2009, el ingeniero Yeen Hernández Hernández, trabajador del Centro de Ingeniería Genética y Biotecnología, llevó a cabo la aplicación del método de estudio de la gestión de la producción propuesto por Acevedo Suárez et al. (2002) a un sistema productivo de medicamentos, teniendo como similitudes, con el procedimiento propuesto, la 
determinación y evaluación de las exigencias técnico-organizativas y de los principios de gestión de producción, determinando así las principales causas que afectaban el aprovechamiento de las capacidades en el sistema productivo de medicamentos. Aunque no proyectaba las posibles soluciones ni determinaba el sistema más factible a aplicar, dicho aspecto es de vital importancia al momento de reorganizar el sistema de producción para buscar mayor capacidad y eficiencia en el flujo del proceso, los cuales son elementos claves en los sistemas actuales y que aparecen reflejados en el procedimiento propuesto.

\section{CONCLUSIONES}

El análisis teórico realizado, en torno a la gestión de producción, permitió disponer de un referente apropiado para sustentar el estudio y dar solución al problema de la investigación identificado y con ello corroborar la hipótesis planteada, aun cuando los análisis referentes a la gestión de producción y sus métodos de estudio en el sistema empresarial cubano son insuficientes.

Se elaboró un procedimiento para la gestión de la producción teniendo en cuenta todos los factores fundamentales que inciden sobre este con el objetivo de mejorar la competitividad, eficiencia y eficacia del proceso productivo integrado de esta entidad. El método de expertos corroboró la factibilidad de la aplicación del procedimiento en la entidad objeto de estudio y su socialización con las demás Unidades Empresariales de Base (UEB) de la empresa.

\section{REFERENCIAS}

Acevedo Suárez, J. A., Gómez Acosta, M. I., Urquiaga Rodríguez, A. J., y Hernández Torres, M. (2002). Organización de la producción y los servicios. La Habana: Ediciones CUJAE.

Araúzo Araúzo, J. A., de Benito Martín, J. J., del Olmo Martínez, R., y Sanz Angulo, P. A. (2004). Situación actual y expectativas de los sistemas de fabricación basados en agentes en el VIII Congreso de Ingeniería de Organización, Leganés, 9-10 de septiembre (pp. 1043-1052). Madrid: Asociación para el Desarrollo de la Ingeniería de Organización. Recuperado de http://www.adingor.es/Documentacion/ClO/ cio2004/comunicaciones/1043-1052.pdf

Ballou, R. (1991). Logística empresarial, control y planificación. España: Díaz de Santos.

Batista, M. (2007). Evaluación del desempeño empresarial a partir de la organización de los procesos en la fábrica de baldosas de Holguín (tesis de pregrado). Universidad de Holguín, Cuba. 
Cespón Castro, R., Ibarra Mirón, S., y Marrero Delgado, F. (septiembre del 2005). Selección del sistema de gestión de la producción en el IX Congreso de Ingeniería de Organización, Gijón, 8 y 9 de septiembre. Madrid: Asociación para el Desarrollo de la Ingeniería de Organización. Recuperado de http://www.adingor.es/ Documentacion/CIO/cio2005/items/ponencias/152.pdf

Decreto Ley $n .^{\circ} 252$, Continuidad y fortalecimiento del Sistema de Dirección y Gestión Empresarial Cubano. (18 de febrero del 2013). Recuperado del archivo digital de la Gaceta Oficial de la República de Cuba https://www.gacetaoficial.gob.cu/ sites/default/files/go_o_007_2013.pdf

Fundora Miranda, A., Taboada Rodríguez, C., Cuellar, H., Urquiaga Rodríguez, A., y Sánchez Lara, A. (1987). Organización y planificación de la producción (t. II). La Habana: Editora ISPJAE.

Hernández Hernández, Y. (2009). Aplicación del método de estudio de la gestión de la producción a un sistema productivo de medicamentos. Ingeniería Industrial, 30(1), 1-8. https://www.redalyc.org/pdf/3604/360433568003.pdf

Lefcovich, M. L. (2004). Sistema Matricial de Control Interno. Una forma eficaz de prevenir y corregir errores. Brasil: El Cid Editor.

Partido Comunista de Cuba. (2016). Actualización de los Lineamientos de la Política Económica y Social del Partido y la Revolución para el período 2016-2021. Recuperado de http://bohemia.cu/wp-content/uploads/2016/09/01Folleto.Lineamientos-4.pdf

Pérez Campaña, M. (2005a). Contribución al control de gestión en elementos de la cadena de suministro. Modelo y procedimientos para organizaciones comercializadoras (tesis doctoral). Universidad Central Marta Abreu, Cuba.

Pérez Campaña, M. (2005b). De la gestión de la producción a la gestión de la cadena de suministros. Recuperado de https://www.monografias.com/trabajos21/gestionproduccion/gestion-produccion.shtml

Portuondo Pichardo, F. M. (1990). Economía de empresas industriales. La Habana: Editorial Pueblo y Educación.

Sipper, D., y Bulfin Jr., R. L. (2009). Planeación y control de la producción. México D. F.: McGraw-Hill.

Torres Cabrera, L., y Urquiaga Rodríguez, A. (2007). Fundamentos teóricos sobre gestión de producción. La Habana: Editorial Félix Varela. 